\title{
Whole genome sequencing of enriched chloroplast DNA using the Illumina GAll platform
}

\author{
Robin A Atherton ${ }^{1,2^{*}+}$, Bennet J McComish ${ }^{1,2+}{ }^{\text {, Lara D Shepherd }}{ }^{1}$, Lorraine A Berry ${ }^{3}$, Nick W Albert ${ }^{1}$, \\ Peter J Lockhart ${ }^{4}$
}

\begin{abstract}
Background: Complete chloroplast genome sequences provide a valuable source of molecular markers for studies in molecular ecology and evolution of plants. To obtain complete genome sequences, recent studies have made use of the polymerase chain reaction to amplify overlapping fragments from conserved gene loci. However, this approach is time consuming and can be more difficult to implement where gene organisation differs among plants. An alternative approach is to first isolate chloroplasts and then use the capacity of high-throughput sequencing to obtain complete genome sequences. We report our findings from studies of the latter approach, which used a simple chloroplast isolation procedure, multiply-primed rolling circle amplification of chloroplast DNA, Illumina Genome Analyzer II sequencing, and de novo assembly of paired-end sequence reads.

Results: A modified rapid chloroplast isolation protocol was used to obtain plant DNA that was enriched for chloroplast DNA, but nevertheless contained nuclear and mitochondrial DNA. Multiply-primed rolling circle amplification of this mixed template produced sufficient quantities of chloroplast DNA, even when the amount of starting material was small, and improved the template quality for Illumina Genome Analyzer II (hereafter Illumina GAII) sequencing. We demonstrate, using independent samples of karaka (Corynocarpus laevigatus), that there is high fidelity in the sequence obtained from this template. Although less than $20 \%$ of our sequenced reads could be mapped to chloroplast genome, it was relatively easy to assemble complete chloroplast genome sequences from the mixture of nuclear, mitochondrial and chloroplast reads.
\end{abstract}

Conclusions: We report successful whole genome sequencing of chloroplast DNA from karaka, obtained efficiently and with high fidelity.

\section{Background}

Chloroplast genomes provide a wealth of information for studies in molecular ecology and evolution. Their conservative gene content and organisation have enabled researchers to isolate homologous loci for comparative studies over different evolutionary time-scales [1-7].

Obtaining the DNA sequence for chloroplast genomes can be achieved by using the polymerase chain reaction (PCR) to amplify chloroplast DNA fragments from genomic DNA (gDNA) extracts. However, this can involve up to 35 amplifications of overlapping chloroplast DNA PCR products $[2,8]$. While this approach is

\footnotetext{
* Correspondence: r.a.atherton@massey.ac.nz

† Contributed equally

${ }^{1}$ Institute of Molecular BioSciences, Massey University, Private Bag 11 222,

Palmerston North, 4442, New Zealand

Full list of author information is available at the end of the article
}

time consuming [8], it has been preferred over protocols that attempt to first separate chloroplasts from other cellular material. Reasons for this appear to be that chloroplast isolation can be troublesome in some species [9] and because rapid chloroplast isolation protocols often produce template which is still contaminated by large quantities of nuclear DNA [10]. Nevertheless, given the depth of sequencing coverage with the Illumina GAII sequencing platform, we were interested to investigate whether this alternative approach could be used for sequencing whole chloroplast genomes without the need for whole genome PCR amplification. Here we report findings which demonstrate that, even with small amounts of chloroplast DNA, and in the presence of large amounts of nuclear DNA, Illumina short read sequencing provides a practical approach for obtaining complete chloroplast genome sequences.

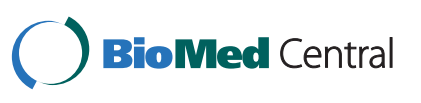




\section{Methods}

\section{Chloroplast isolation}

Fresh leaf material was obtained from two cultivated karaka trees originating in Rekohu/Chatham Islands and the Kermadec Islands, New Zealand. Leaf material was collected and processed immediately for the sample from the Chatham Islands and within $3 \mathrm{~h}$ for the sample from the Kermadec Islands. Leaf samples weighing 2.5-5 g were excised from living trees and processed as follows. Chloroplasts were isolated using a protocol originally designed for isolating chloroplasts from Arabidopsis thaliana [11] with minor modifications: (i) the leaf material was homogenised using an Ultra-Turrax homogeniser with an N18 rotor (Janke \& Kunkel IKA, Hamburg, Germany); (ii) the homogenate was passed through a double layer of washed and autoclaved nappy (diaper) liner (Johnson \& Johnson Ltd.) rather than through Miracloth (Calbiochem); and (iii) the final centrifugation step was carried out using a Sorvall SS32 angled rotor, rather than a swinging bucket rotor. After the final centrifugation step, DNA was extracted from pooled chloroplasts for each tree sample using a DNEasy Plant Mini Kit (Qiagen) following the manufacturer's instructions. Genomic DNA (gDNA) was extracted from silica-dried karaka leaf material from the same accessions using a DNEasy Plant Mini Kit (Qiagen).

\section{Multiply-primed rolling circle amplification}

Multiply-primed rolling circle amplification (RCA) was used to produce an abundance of purified chloroplast DNA template in preparation for sequencing [12]. This technique involves isothermal, strand-displacing amplification using multiple primers and is capable of yielding a large amount of product from very little starting DNA template [13]. Phi29, the DNA polymerase used in multiply-primed RCA, is reported to have a very low level of amplification bias making the template suitable for whole genome sequencing [14]. Chloroplast-enriched DNA (cpDNA) from both karaka samples was amplified in this way using a REPLI-g ${ }^{\mathrm{TM}}$ Mini Kit (Qiagen) following the manufacturer's instructions, with the exception that samples were incubated at room temperature for $9 \mathrm{~min}$ rather than the recommended $3 \mathrm{~min}$. This extension time consistently produced better results with different plant samples. The kit produced $\sim 5 \mu$ g of product for each sample.

\section{Confirmation of chloroplast DNA enrichment}

Genomic DNA (gDNA), chloroplast-enriched DNA (cpDNA) and RCA amplified chloroplast-enriched DNA (RCAcpDNA) from the Chatham Island sample were quantified fluorometrically using the Quant-iT $\mathrm{T}^{\mathrm{TM}} \mathrm{dsDNA}$ HS assay kit on a Qubit ${ }^{\text {TM }}$ Quantitation Platform (Invitrogen). The concentration of the gDNA, cpDNA and
RCAcpDNA was $110 \mathrm{ng} \mu \mathrm{L}^{-1}, 20 \mathrm{ng} \mu \mathrm{L}^{-1}$ and $104 \mathrm{ng} \mu \mathrm{L}^{-1}$, respectively, in a total volume of $50 \mu \mathrm{L}$ of $\mathrm{AE}$ buffer (Qiagen). The purity of gDNA, cpDNA and RCAcpDNA samples was determined by $A_{260} / A_{280}$ and $A_{260} / A_{230}$ ratios on a NanoDrop (NanoDrop Technologies) spectrophotometer. Enrichment for chloroplast DNA was determined by quantitative real-time PCR (qPCR) with gDNA, cpDNA and RCAcpDNA templates; the quantity of the plastid gene $p s b B$ was determined relative to nuclear encoded $18 \mathrm{~S} r R N A$ by comparative quantification [15]. Gene-specific primers were designed for $p s b B$ ( $p s b B$ F 5'GGGGGTTGGAGTATCACAGG3'; $p s b B$ R 5'CCAAGAAGCACAAGCCAGAA3', 103 bp amplicon) using Primer3 [16] and primers for $18 \mathrm{~S}$ are described by Zhu and Altmann [17]. qPCR was performed using Lightcycler480 SYBR Green1 Master (Roche Diagnostics) reagents in a Rotor Gene 3000 instrument (Corbett Research) with four technical replicates per sample. Template DNA was diluted 20-fold for cpDNA, and 100 -fold for gDNA and RCAcpDNA samples for qPCR. The qPCR cycling conditions were: $95^{\circ} \mathrm{C} 10 \mathrm{~min},\left(95^{\circ} \mathrm{C}\right.$ $\left.10 \mathrm{~s}, 60^{\circ} \mathrm{C} 15 \mathrm{~s}, 72^{\circ} \mathrm{C} 20 \mathrm{~s}\right) \times 40$ cycles with fluorescent detection at $72^{\circ} \mathrm{C}$ and during the final melt. Melt curve analysis confirmed the amplification of a single product.

\section{Illumina GAll sequencing}

The RCAcpDNA samples from both accessions were sequenced by Massey Genome Service (Massey University, Palmerston North, New Zealand). A 75 bp pairedend run was performed on the Illumina GAII with the two samples described here in a single lane along with four other samples from a separate experiment. Samples were prepared for sequencing as follows: genomic DNA libraries were prepared by fragmenting purified genomic DNA using a nebulisation kit (Invitrogen), paired-end index adaptor ligation (Illumina) and 18 cycles of PCR enrichment using the Illumina Paired-End Genomic DNA library preparation kit, Illumina Multiplex Oligonucleotide library preparation kit and Illumina Multiplex Paired-End Genomic DNA library preparation protocol. The enriched libraries were quantified using an ND-1000 NanoDrop spectrophotometer (NanoDrop Technologies) and quality checked by Agilent 2100 Bioanalyzer, DNA 1000 Labchip kit assay. The libraries were then diluted to a $10 \mathrm{nM}$ concentration using EB buffer (Qiagen) and quantified for optimal cluster density using the LightCycler 480 system Absolute Quantification protocol (Roche Diagnostics) and the LightCycler ${ }^{\circledR} 480$ SYBR Green I Master kit (Roche Diagnostics). The libraries were pooled at equal molarity and amplified in one flow cell lane on the Illumina Cluster Station instrument at a density of 140,000 clusters per tile and a molarity of 13 pM using the Illumina Paired- 
End Cluster Generation kit v2. The amplified libraries were sequenced on the Illumina GAII instrument using 4 Illumina 36 cycle SBS sequencing kits (v3), Illumina Multiplex Sequencing Primers and PhiX control kit v2, on a 75 bp paired-end indexed run. After sequencing, the resulting images were analysed with the proprietary Illumina pipeline v1.3. Reads for each of the indexed samples were then separated using a custom Perl script.

\section{Assembly}

Reads from each indexed sample were trimmed to remove poor quality sequence at the 3 ' end. To determine the optimum trim length, initial de novo assemblies were made for read sets of different length (untrimmed reads, and reads trimmed to 70, 65, 55, 50 bp). These assemblies were carried out using Velvet 0.7 [18] with a range of hash lengths from 33 to 63 and a minimum k-mer coverage of $5 \times$. For these initial assemblies, the data were treated as single reads, that is, the paired-end information was not used. Maximum contig lengths and N50 values were tabulated and the hash lengths that gave the highest N50 for each trimmed set of reads were selected for further optimisation. A second round of assembly was carried out on each trimmed set of reads using the hash length determined above and varying the coverage cut-off parameter from 1 to 100 . Finally, paired-end assembly was carried out for each of these read-length/hash-length combinations using the coverage cut-off value that gave the highest N50 value. For these paired-end assemblies, expected coverage was set to the length-weighted median of the coverage values obtained in the initial single read assemblies, and the insert length was estimated as $240 \mathrm{bp}$. Assembled contigs were aligned to the Cucumis sativus chloroplast genome [GenBank: NC_007144; GenBank: DQ119058] using Geneious 4.7 [19].

Four short regions of ambiguous sequence were checked by PCR amplification using the following primers, custom designed using Primer3 [16] unless referenced: Corlaerps2-rpoc2F (TATAGGGTGCCATTCG AGGA), Corlaerps2-rpoc2R GTATCAACAACGGCCAATCC; CorlaendhAF (GGAATAGGATGGAGATAAGAAAGAC), CorlaendhAR (CACGATTCCG ATCCAGAGTA); psbJ ATAGGTACTGTARCYGGTAT [20], petA AACARTTYGARAAGGTTCAATT [20]; psbAR (CGCGTCTCTCTAAAATTGCAGTCAT) [21], CorlaepsbA-R (ATCCGACTAGTTCCGGGTTC). Figure 1 shows the relative position of the priming sites on the karaka chloroplast genome. The PCR cycling conditions were modified slightly from an existing published protocol [20] as follows: template denaturation at $80^{\circ} \mathrm{C}$ for 5 min followed by 32 cycles of denaturation at $95^{\circ} \mathrm{C}$ for $1 \mathrm{~min}$, primer annealing at $50^{\circ} \mathrm{C}$ for $1 \mathrm{~min}$, followed by a ramp of $0.3^{\circ} \mathrm{C} / \mathrm{s}$ to $65^{\circ} \mathrm{C}$, and primer extension at $65^{\circ} \mathrm{C}$ for $4 \mathrm{~min}$; followed by a final extension step of $5 \mathrm{~min}$ at $65^{\circ} \mathrm{C}$. Amplified PCR products were sequenced using the BigDye Terminator Cycle Sequencing Kit (Applied Biosystems) and an ABI 3730 automated capillary sequencer at Massey Genome Service (Massey University, Palmerston North, New Zealand). The resulting sequences were visualised and edited using Sequencher 4.9 software for Mac (Gene Codes Corporation, Ann Arbor, MI). Using Geneious [19], the four ambiguous regions of the assembled genome were edited, where necessary, to match the Sanger sequences.

\section{Mapping and annotation}

In order to check the de novo assembly, reads were aligned against the assembled genome using BWA [22] with default parameters. Only $19.6 \%$ of reads were successfully aligned, but this was sufficient to give a mean coverage of $400 \times$. This mapping enabled us to resolve some short regions of ambiguous sequence in the assembly. The final complete chloroplast genome sequence was annotated using DOGMA [23] and through comparison to published complete chloroplast genome sequences available through GenBank [24].

\section{Results}

\section{DNA sequencing template for karaka}

The relative quantity of chloroplast DNA in samples of total gDNA, cpDNA and RCAcpDNA preparations was determined by quantitative PCR (Figure 2). The enriched cpDNA sample had 2.6-fold higher levels of chloroplast DNA compared to a standard gDNA preparation prior to RCA and 2.2-fold after RCA. The purity of the DNA preparations was assessed by spectrophotometric $\mathrm{A}_{260} / \mathrm{A}_{280}$ and $A_{260} / A_{230}$ ratios. The gDNA and cpDNA samples had low ratios, indicating the presence of protein $\left(\mathrm{A}_{260} /\right.$ $\mathrm{A}_{280}=1.66$ and 1.69 , respectively) and other contaminants such as carbohydrates and phenolics $\left(\mathrm{A}_{260} / \mathrm{A}_{230}=\right.$ 1.49 and 1.28 , respectively). RCA of the cpDNA-enriched sample substantially increased the quantity and quality of template DNA $\left(\mathrm{A}_{260} / \mathrm{A}_{280}=1.75, \mathrm{~A}_{260} / \mathrm{A}_{230}=2.20\right)$.

\section{Sequencing and assembly of the karaka chloroplast genomes}

Paired-end sequencing of the RCAcpDNA template in a single lane on an Illumina GAII flow cell produced 1.84 and 1.76 million reads for the Chatham Islands sample and Kermadec Islands sample respectively. The Chatham Islands sample was assembled de novo as described in the methods section. The Kermadec Island sample was then mapped to this assembly.

The most useful assembly was achieved for the Chatham Islands sample, with reads trimmed to $50 \mathrm{bp}$, coverage cut-off of 9 and expected coverage of 40 . While 


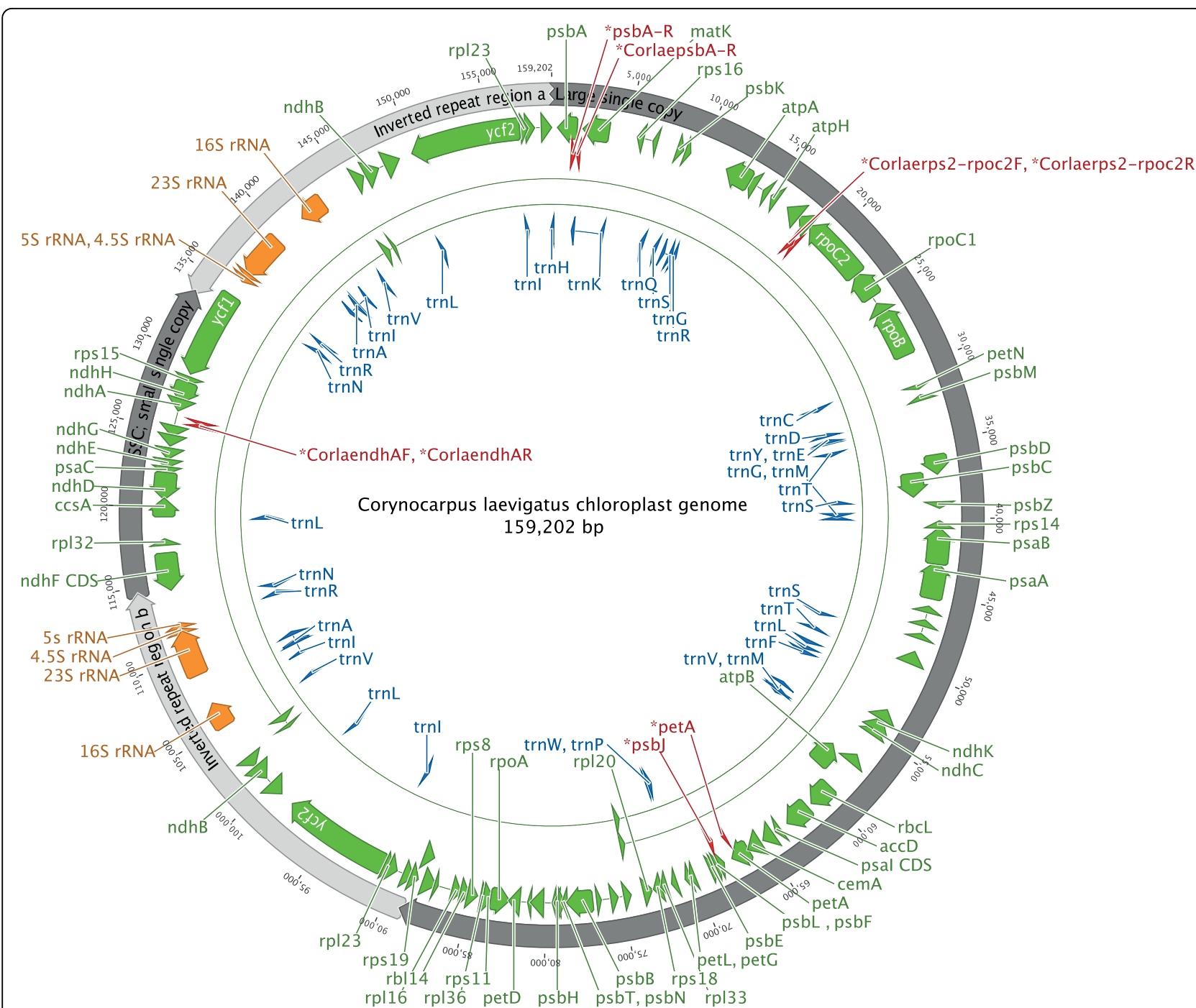

Figure 1 Complete gene map of the entire Corynocarpus laevigatus chloroplast genome. Arrowheads depict the direction of transcription. Binding sites for the primers used to generate the PCR products for resolving homopolymers are indicated in red. This figure was produced using Geneious [19].

some of our other assemblies had higher overall N50 values or longer maximum contig lengths, these improved statistics did not reflect any real improvement in the assembly, as the large single-copy region was merged with part of the inverted repeat to form a single long contig at the expense of a more fragmented assembly of the remainder of the inverted repeat and small single-copy region.

The optimal assembly parameters produced a total of 13 contigs, four of which could be mapped to the Cucumis sativus chloroplast genome. These four contigs ranged from $7,857 \mathrm{bp}$ to $88,955 \mathrm{bp}$ in length and covered the entire chloroplast genome. The nine remaining contigs were much shorter, ranging from $81 \mathrm{bp}$ to $669 \mathrm{bp}$. These were checked against the GenBank nucleotide database using the web-based BlastN algorithm [25], and the only significant alignments found were to nuclear ribosomal DNA sequences.

Of the four contigs mapping to the C. sativus chloroplast genome, one mapped to the inverted repeat region, one to the large single-copy region, and two to the small single-copy region. The overlaps between contigs at all four junctions between inverted repeat and single-copy regions were $40 \mathrm{bp}$ long, indicating that contig extension was interrupted by the ambiguity of the overlap rather than by insufficient coverage. The overlap between the two contigs that formed the small singlecopy region consisted of a polyA-polyT homopolymer. The contig corresponding to the large single-copy region contained six short (1-49 bp) stretches of 


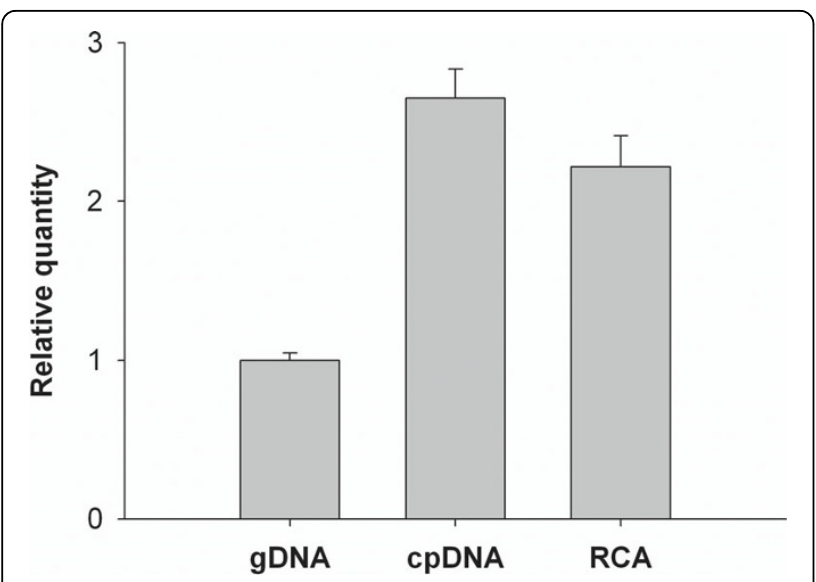

Figure 2 Enrichment of chloroplast DNA from karaka. The relative quantity of chloroplast DNA was determined in preparations of genomic DNA (gDNA), chloroplast-enriched DNA (cpDNA) and RCA amplified chloroplast-enriched cpDNA (RCA). Quantitative PCR amplification of the plastid encoded $p s b B$ gene was compared to amplification of nuclear 185 rRNA. The mean of four technical replicates \pm SE has been reported as fold-differences relative to gDNA.

ambiguous bases where Velvet was unable to resolve the sequence due to mono- or dinucleotide repeats. Three of these stretches were resolved by mapping the reads to the assembled sequence as described in the methods. The other three stretches, along with the overlap between the two contigs that made up the small singlecopy region, were checked by PCR amplification and Sanger sequencing.

The final assembled chloroplast genome sequence (shown in Figure 1) was checked by mapping the original Illumina reads against the assembled sequence. A total of 344,475 of 1.76 million reads (19.6\%) were successfully aligned, suggesting that approximately $80 \%$ of the DNA sequenced was of nuclear or mitochondrial origin.

\section{Discussion}

We have shown that the modified chloroplast isolation protocol produced DNA template sufficiently enriched for chloroplast sequence to allow de novo assembly of the chloroplast genome. Comparison of the two genomes indicated high fidelity with less than $0.002 \%$ error. Whilst RCA of the cpDNA marginally reduced the final ratio of cpDNA/gDNA in the enriched sample, the purity of the DNA was of a higher quality for Illumina sequencing.

The coverage cut-off parameter of the Velvet assembler was crucial for successful assembly, as it allowed the chloroplast sequence reads to be assembled without interference from nuclear sequence. Although over $80 \%$ of reads failed to align to our assembled chloroplast genome, and are likely to be of nuclear origin, the much greater size of the nuclear genome means that these reads were present at much lower coverage than the chloroplast reads. A notable exception is nuclear ribosomal DNA, which is present in many copies in the nuclear genome, thus its coverage was comparable to that of the chloroplast genome in our enriched sample.

The lower copy number of the nuclear genome compared to the chloroplast genomes means that nuclear copies of chloroplast DNA sequences are very unlikely to affect our assemblies. In contrast, nuclear-encoded chloroplast DNA may be more difficult to distinguish from chloroplast-encoded sequences if amplified by chloroplast DNA primers. Thus, this is potentially another advantage of the approach we have used for determining complete chloroplast genome sequences.

Finally, although de novo assembly was a feature of our protocol, the availability of a related reference genome did help with our final assembly, allowing us to separate contigs derived from chloroplast DNA from the few short contigs of nuclear origin. This was helpful for determining the arrangement of chloroplast contigs.

\section{Conclusions}

We have successfully applied a whole genome sequencing approach to determine the complete chloroplast genome sequence of karaka. We have also applied this approach more recently to a range of New Zealand seed plants (gymnosperms and angiosperms: herbaceous and woody plants), sequencing up to three chloroplast genomes per GAII flow cell lane. Thus we are confident that the approach that we describe here for karaka provides a fast and efficient protocol for obtaining whole chloroplast genome sequences for seed plants.

The fully annotated chloroplast genome sequence of karaka (Corynocarpus laevigatus) from the Chatham Islands sample has been deposited in the GenBank database under accession number HQ207704.

\section{Acknowledgements}

We would like to acknowledge the help and support of the Hokotehi Moriori Trust Board of Rekohu/Chatham Islands and Ngāti Kuri and Te Aupouri, tangata whenua (indigenous inhabitants) of the Kermadec Islands. We would also like to acknowledge Te Ati Awa (Taranaki Whānui) in the Wellington and Hutt Valley regions of New Zealand, with whom we have ongoing consultations. We thank Peter de Lange (Department of Conservation), Rewi Elliot and Eleanor Burton (Otari Wilton's Bush, Wellington) for assistance with sample collection. Samples were collected under Department of Conservation permit WA-23814-FLO and Otari Wilton's Bush permit 145. Thanks also to Maurice Collins (MGS) for pipeline analysis, Trish McLenachan (IMBS, Massey University) and Jan Binnie (IMBS, Massey University) for laboratory assistance. This study was funded by the New Zealand Marsden Fund (contract numbers MAU050; MAU0709) and the Allan Wilson Centre for Molecular Ecology and Evolution. Robin Atherton acknowledges financial support from a Massey University Doctoral Scholarship, JP Skipworth Scholarship for Plant Biology, Hokotehi Moriori Trust Board, Intellectual Property in Cultural Heritage (IPinCH) and the Allan Wilson Centre for Molecular Ecology and Evolution. Bennet McComish 
acknowledges support from an Allan Wilson Centre for Molecular Ecology and Evolution Doctoral Scholarship. Peter Lockhart is supported by a James Cook Research Fellowship from the Royal Society of New Zealand.

\section{Author details}

${ }^{1}$ Institute of Molecular BioSciences, Massey University, Private Bag 11 222, Palmerston North, 4442, New Zealand. ${ }^{2}$ Allan Wilson Centre for Molecular Ecology and Evolution, Massey University, Private Bag 11 222, Palmerston North, 4442, New Zealand. ${ }^{3}$ Massey Genome Service, Massey University, Private Bag 11 222, Palmerston North, 4442, New Zealand. ${ }^{4}$ Institute of Fundamental Sciences, Massey University, Private Bag 11 222, Palmerston North, 4442, New Zealand.

\section{Authors' contributions}

RAA helped with the design of the study, isolated chloroplasts, extracted the DNA, performed RCA amplification of cpDNA, carried out PCR experiments and submitted data to GenBank, as well as drafting, contributing to and editing the manuscript; BJM carried out de novo assembly and annotation of the genome, contributed to and edited the manuscript; LDS contributed to, edited and revised several versions of the manuscript and provided academic guidance to RAA during her PhD study. LB carried out sample preparation and sequencing on the Illumina GAll; NWA designed and performed GPCR assays and contributed to and edited the manuscript; PJL conceived of and designed the study, contributed to, edited and revised several versions of the manuscript and provided academic guidance to RAA during her PhD study. All authors read and approved the final manuscript.

\section{Competing interests}

The authors declare that they have no competing interests.

Received: 25 June 2010 Accepted: 28 September 2010

Published: 28 September 2010

\section{References}

1. Gruenheit N, Lockhart PJ, Steel M, Martin W: Difficulties in testing for covarion-like properties of sequences under the confounding influence of changing proportions of variable sites. Molecular Biology and Evolution 2008, 25(7):1512-1520.

2. Goremykin W, Hirsch-Ernst Kl, Wolfl S, Hellwig FH: Analysis of the Amborella trichopoda chloroplast genome sequence suggests that Amborella is not a basal angiosperm. Molecular Biology and Evolution 2003, 20(9):1499-1505.

3. Knapp M, Stockler K, Havell D, Delsuc F, Sebastiani F, Lockhart PJ: Relaxed molecular clock provides evidence for long-distance dispersal of Nothofagus (southern beech). Plos Biology 2005, 3(1):38-43.

4. Stehlik I, Blattner FR, Holderegger R, Bachmann K: Nunatak survival of the high Alpine plant Eritrichium nanum (L.) Gaudin in the central Alps during the ice ages. Molecular Ecology 2002, 11(10):2027-2036.

5. Ingvarsson PK, Ribstein S, Taylor DR: Molecular evolution of insertions and deletion in the chloroplast genome of Silene. Molecular Biology and Evolution 2003, 20(11):1737-1740.

6. Golenberg EM, Clegg MT, Durbin ML, Ma DP: Evolution of a noncoding region of the chloroplast genome. Molecular Phylogenetics and Evolution 1993, 2(1):13.

7. Powell W, Morgante M, Andre C, Mcnicol JW, Machray GC, Doyle رل Tingey SV, Rafalski JA: Hypervariable microsatellites provide a general source of polymorphic DNA markers for the chloroplast genome. Current Biology 1995, 5(9):1023-1029.

8. Cronn R, Liston A, Parks M, Gernandt D, Shen R, Mockler T: Multiplex sequencing of plant chloroplast genomes using Solexa sequencing-bysynthesis technology. Nucleic Acids Research 2008, 36(19):e122-e122.

9. Dhingra A, Folta KM: ASAP: Amplification, sequencing \& annotation of plastomes. BMC Genomics 2005, 6:176.

10. Jansen RK, Raubeson LA, Boore JL, DePamphilis CW, Chumley TW, Haberle RC, Wyman SK, Alverson AJ, Peery R, Herman SJ, et al: Methods for obtaining and analyzing whole chloroplast genome sequences. Molecular Evolution: Producing the Biochemical Data, Part B 2005, 395:348-384.

11. Aronsson $H$, Jarvis P: A simple method for isolating import-competent Arabidopsis chloroplasts. FEBS Letters 2002, 529(2-3):215-220.

12. Jansen R, Raubeson L, Boore J, dePamphilis C, Chumley T, Haberle R, Wyman S, Alverson A, Peery R, Herman S, et al: Methods for obtaining and analyzing whole chloroplast genome sequences. In Methods in Enzymology: Molecular Evolution: Producing the Biochemical Data, Part B. Edited by: Zimmer E, Roalson E. San Diego: Elsevier Academic Press Inc; 2005:348-384:896.

13. Dean FB, Nelson JR, Giesler TL, Lasken RS: Rapid amplification of plasmid and phage DNA using phi29 DNA polymerase and multiply-primed rolling circle amplification. Genome Research 2001, 11(6):1095-1099.

14. Dean FB, Hosono S, Fang LH, Wu XH, Faruqi AF, Bray-Ward P, Sun ZY, Zong QL, Du YF, Du J, et al: Comprehensive human genome amplification using multiple displacement amplification. Proceedings of the National Academy of Sciences of the United States of America 2002, 99(8):5261-5266

15. Pfaffl MW: A new mathematical model for relative quantification in realtime RT-PCR. Nucleic Acids Research 2001, 29(9):e45.

16. Rozen S, Skaletsky HJ: Primer3 on the www for general users and for biologist programmers. In Bioinformatics Methods and Protocols: Methods in Molecular Biology. Edited by: Krawetz S, Misener S. Totowa, NJ: Humana Press; 2000:365-386.

17. Zhu L, Altmann SW: mRNA and 18S-RNA coapplication-reverse transcription for quantitative gene expression analysis. Anal Biochem 2005, 345:102-109

18. Zerbino D, Birney E: Velvet: Algorithms for de novo short read assembly using de Bruijn graphs. Genome Research 2008, 18(5):821-829.

19. Drummond AJ, Ashton B, Cheung M, Heled J, Kearse M, Moir R, StonesHavas S, Thierer T, Wilson A: Geneious v4.7. 2009 [http://www.geneious. com].

20. Shaw J, Lickey E, Schilling E, Small R: Comparison of whole chloroplast genome sequences to choose noncoding regions for phylogenetic studies in angiosperms: the tortoise and the hare III. American Journal of Botany 2007, 94(3):275.

21. Winkworth RC, Grau J, Robertson A, Lockhart PJ: The origins and evolution of the genus Myosotis L. (Boraginaceae). Molecular Phylogenetics and Evolution 2002, 24(2):180-193.

22. Li H, Durbin R: Fast and accurate short read alignment with BurrowsWheeler transform. Bioinformatics 2009, 25(14):1754-1760.

23. Wyman SK, Jansen RK, Boore JL: Automatic annotation of organellar genomes with DOGMA. Bioinformatics 2004, 20(17):3252-3255.

24. Benson DA, Karsch-Mizrachi I, Lipman DJ, Ostell J, Sayers EW: GenBank. Nucleic Acids Research 2009, 37:D26-D31

25. Altschul S, Madden T, Schaffer A, Zhang J, Zhang Z, Miller W, Lipman D: Gapped BLAST and PSI-BLAST: a new generation of protein database search programs. Nucleic Acids Research 1997, 25(17):3389.

doi:10.1186/1746-4811-6-22

Cite this article as: Atherton et al: Whole genome sequencing of enriched chloroplast DNA using the Illumina GAll platform. Plant Methods 2010 6:22.

\section{Submit your next manuscript to BioMed Central and take full advantage of:}

- Convenient online submission

- Thorough peer review

- No space constraints or color figure charges

- Immediate publication on acceptance

- Inclusion in PubMed, CAS, Scopus and Google Scholar

- Research which is freely available for redistribution

Submit your manuscript at www.biomedcentral.com/submit
C Biomed Central 